\title{
Quelle ingénierie pour l'intégration du développement durable à l'écotourisme québécois? Le cas de la coopérative de la vallée Bras-du-Nord ${ }^{\mathrm{a}}$
}

\author{
Manu Tranquard, Ph.D., LL.M. \\ Professeur et responsable du programme de baccalauréat en plein air \\ Laboratoire d'Expertise et de Recherche en Plein Air (LERPA) \\ Université du Québec à Chicoutimi
}

\section{INTRODUCTION}

Dans une contribution précédente ${ }^{1}$, il a été observé qu'une ingénierie appropriée pourrait en théorie favoriser la mise en œuvre de critères environnementaux de la durabilité écotouristique, lors de la phase de conception d'un projet écotouristique. Pour appuyer cette analyse et y apporter d'éventuels ajustements et approfondissements, une étude de cas est ici conduite. Cette étude porte sur la Coopérative d'écotourisme de la Vallée Bras-du-Nord et plus spécifiquement sur son projet Tourisme, paysage et coopération. Elle vise à évaluer, sur la base des critères définis précédemment, les conditions de mise en place d'un plan de développement durable.
Cette coopérative de solidarité a été créée en 2002 dans le but d'assurer le développement écotouristique de la région agroforestière de $\mathrm{St}-$ Raymond de Portneuf. Mis en place à partir de 2007, le projet Tourisme, paysage et coopération est un plan de développement durable (DD) du territoire géré par la Coopérative ${ }^{\mathrm{b}}$. Par le biais de ce projet, la Coopérative a pu consolider son modèle d'écotourisme, et mettre sur pied des initiatives qui visent la protection du territoire et la participation de la communauté locale à sa gestion.

\section{Illustration 1 - Situation géographique de la MRC de Portneuf, Québec}

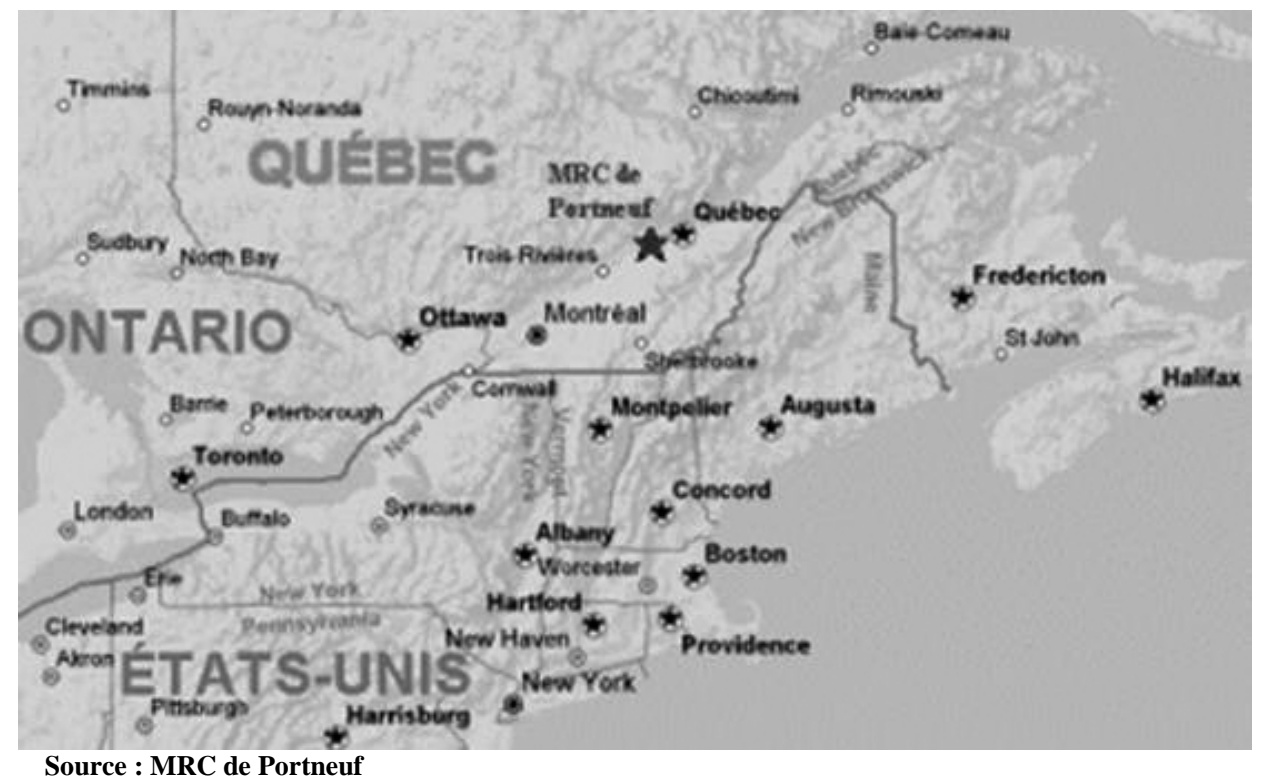




\section{Illustration 2 - Situation géographique de la Vallée Bras-du-Nord}

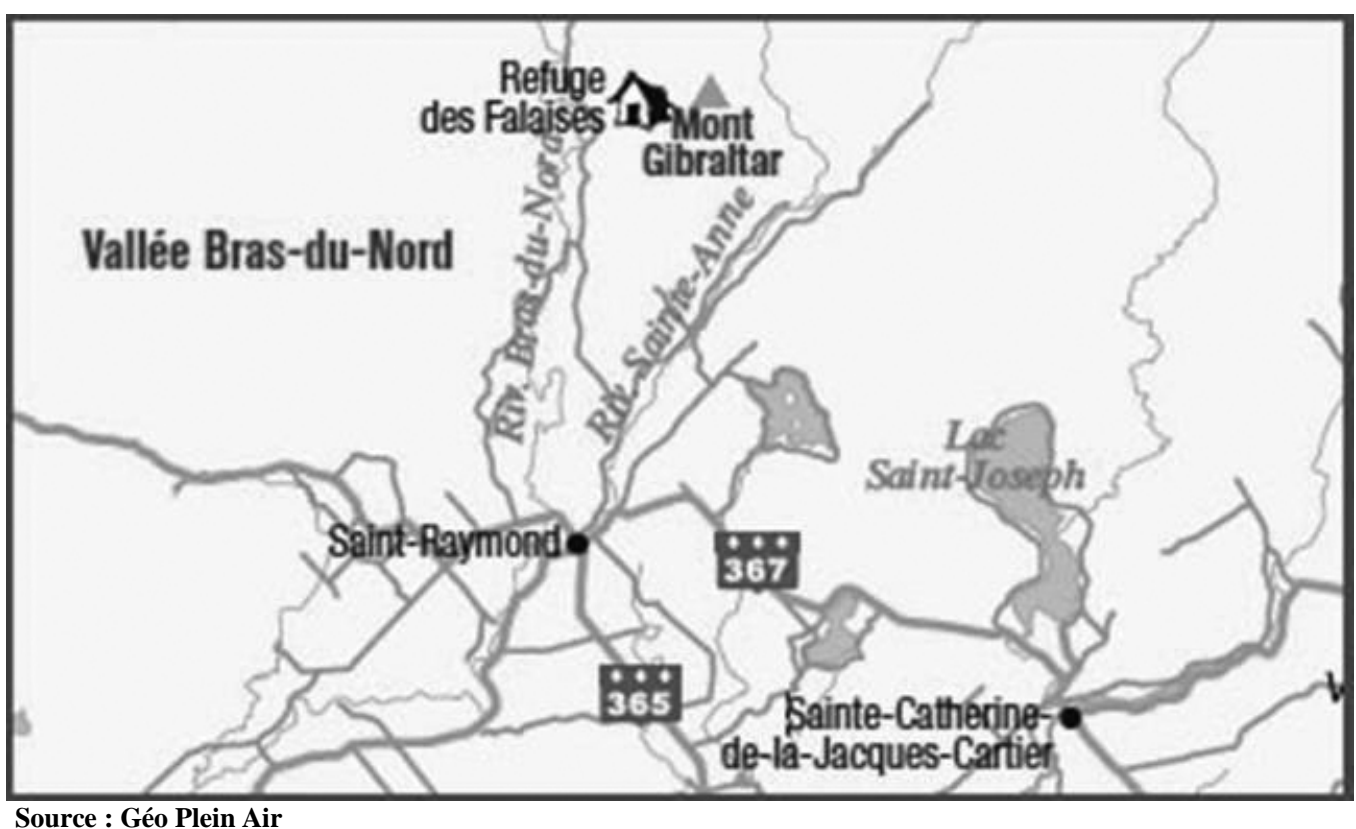

Plusieurs des actions entreprises et des axes d'intervention retenus dans le cadre de ce projet reposent sur l'utilisation de méthodes et d'outils relevant d'une forme d'ingénierie écotouristique. Ce concept réfère aux outils employés de manière scientifique et méthodique comme supports techniques à la réalisation d'opérations complexes. Deux ans après la fin dudit projet, l'évaluation de ses résultats nous permet de dresser un bilan étayé de l'utilisation de cette ingénierie écotouristique versus ses objectifs. Concrètement, la présente évaluation repose sur trois questions : 1) Existe-t-il un lien d'efficience entre l'atteinte des objectifs de développement durable et les outils utilisés dans le cadre d'un projet de développement écotouristique? 2) Le recours à ces outils se réalise-t-il à diverses étapes de la vie du projet? 3) Y a-t-il eu, dans la formulation du projet, une prise en compte de critères territoriaux, tels que les spécificités sociales, politiques, des relations entre parties prenantes ou encore du partenariat entre les acteurs?

La méthodologie retenue pour aborder ces questions repose essentiellement sur une recherche documentaire correspondant aux rapports produits par la Coopérative et les organismes partenaires et subventionnaires. La majorité de ces rapports sont des documents publics, accessibles via le site web de la Coopérative ou auprès d'instances directement impliquées dans le processus de développement durable (ex. : FAQDD, Chambre de commerce, CLD). D'autres sont des données plus techniques, obtenues de la Coopérative dans le cadre de la présente recherche: une entrevue téléphonique avec la direction de la Coopérative, conduite en parallèle à cette recherche documentaire, a permis d'en clarifier certains contenus ${ }^{c}$. L'analyse des résultats du plan d'action de développement durable de la Coopérative de la Vallée Bras-du-Nord a été effectuée en soumettant ces rapports à une grille de critères sur la durabilité, adaptée aux particularismes de l'écotourisme et à son contexte territorial.

Avant de procéder à l'évaluation du cas, il importe d'en justifier le choix et son exemplarité à titre de projet écotouristique et territorial. La démonstration portera également sur la cohérence du plan d'action de développement durable. Par la suite, l'évaluation proprement dite ciblera les modalités de recours à une forme d'ingénierie comme support à ce même plan d'action, et les résultats qui en ont découlé. 


\section{CONTEXTE DE LA NAISSANCE DU PROJET TOURISME, PAYSAGE ET COOPÉRATION}

La formation de la Coopérative de la Vallée Brasdu-Nord repose sur un objectif de développement écotouristique durable. Son organisation et les projets mis sur pied s'inscrivent dans cet objectif. Cinq ans après sa création, le projet Tourisme, paysage et coopération vient le concrétiser un peu plus, en ciblant des objectifs spécifiques issus d'une interprétation et d'une application locale des principes de la Loi $118^{\mathrm{d}}$.

\section{Illustration 3 - Vallée Bras-du-Nord (Portneuf, Québec)}

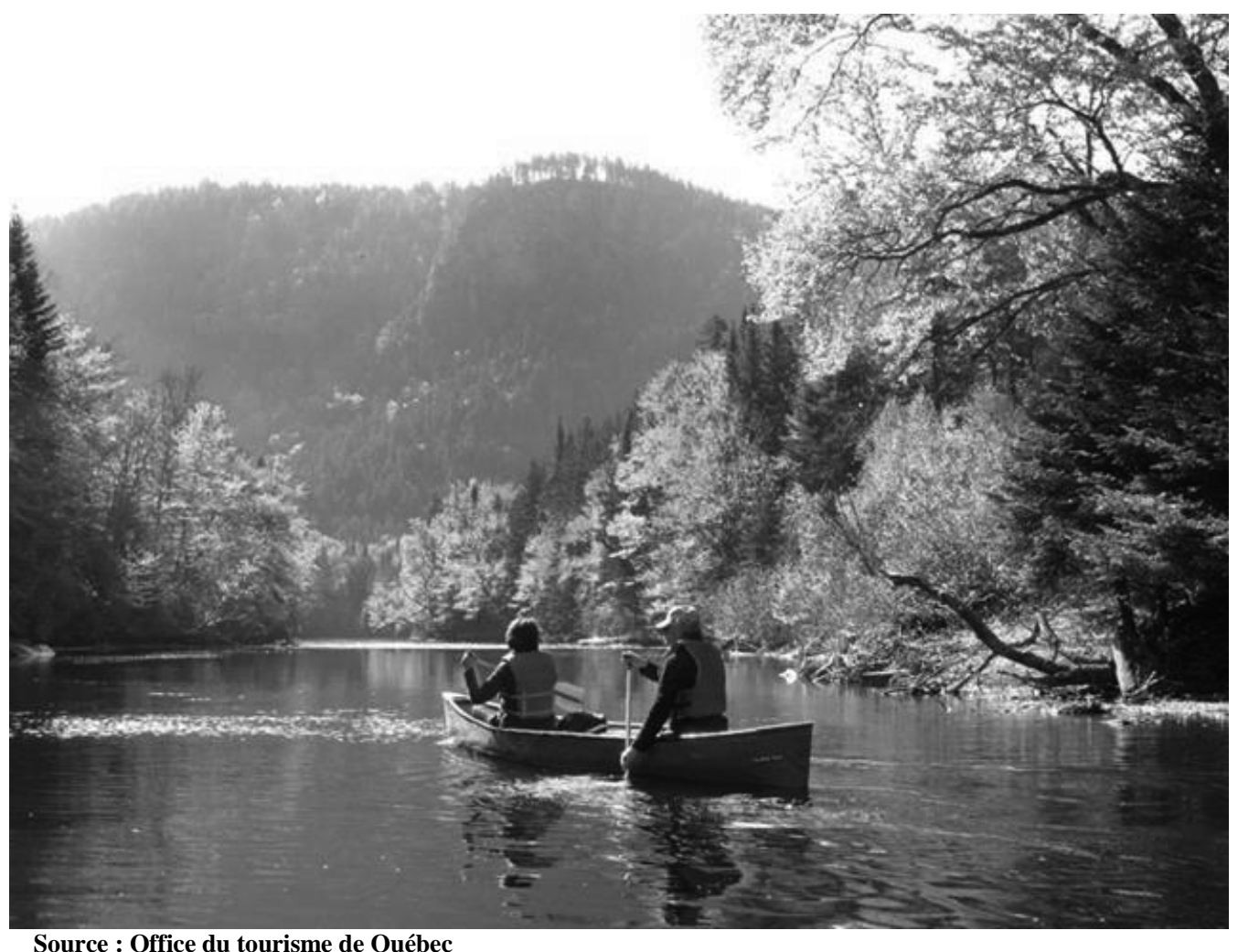

\subsection{Le projet territorial de la Coopérative d'écotourisme de la Vallée Bras-du-Nord (Portneuf)}

La Vallée Bras-du-Nord se situe sur un territoire offrant $35 \mathrm{~km}$ de rivière sinueuse, des parois rocheuses, un rang agricole et un patrimoine paysager et historique rare, le tout réparti sur des terres publiques et privées, incluant notamment un territoire de la Nation huronne-wendat. Le début de son exploitation récréotouristique est marqué par l'accroissement rapide du nombre de villégiateurs aux pratiques néfastes pour l'environnement et le développement local :

$\mathrm{Au}$ cours des années 80 , la présence des villégiateurs citadins se fait de plus en plus remarquer, notamment par le déboisement des rives. Au début des années 90 , les citoyens sonnent l'alarme de l'envahissement indiscipliné des amateurs de plein air. La pratique anarchique de certains sports cause deux décès. Les propriétés privées sont violées. On pratique le camping sauvage... c'est le cas de le dire!

En réaction à cette situation jugée problématique, les intervenants locaux, soit les propriétaires riverains, les entreprises de services récréotouristiques, les travailleurs récréoforestiers et touristiques, la Chambre de commerce et la Corporation de développement se concertent. Ils créent la Coopérative de solidarité Vallée Bras-du-Nord à l'été 2002. Les parties prenantes au projet souhaitent favoriser un développement de qualité, qui 
mettrait en valeur la vallée, ainsi que renforcer leur capacité à protéger l'environnement local par la promotion de l'écotourisme. Dès lors, le développement durable devient le socle fondateur de la Coopérative. Cette Coopérative se donne en effet pour mission d' " assurer, dans un esprit coopératif, un développement récréotouristique durable et harmonieux propice à la mise en valeur de la vallée du Bras-du-Nord et de la région de St-Raymond $»^{\mathrm{e}}$. Le territoire se veut être « un modèle tangible, vivant et inspirant de développement durable ».

\section{L'outil principal destiné à supporter}

l'objectif de développement durable est la formule coopérative. La structure

d'économie sociale retenue, soit la

coopérative de solidarité, intègre la notion de rentabilité, à la fois économique et sociale.

Aux dires des responsables, l'outil principal destiné à supporter l'objectif de développement durable est la formule coopérative. La structure d'économie sociale retenue, soit la coopérative de solidarité, intègre la notion de rentabilité, à la fois économique et sociale. Statutairement, la Coopérative garantit aux membres l'équité dans le partage des résultats: lorsqu'elle réalise des excédents, les trop-perçus peuvent être distribués sous forme de ristournes au prorata des opérations effectuées par chacun des membres et non en fonction de leur participation financière. Les excédents peuvent également être affectés à la réserve de la Coopérative, soit pour consolider sa situation financière, soit pour effectuer du développement. L'entreprise est contrôlée par ses membres. Les usagers d'une coopérative assument ensemble les responsabilités d'entrepreneurs (propriété et contrôle de l'entreprise). Ils se procurent des biens et des services ou y trouvent un emploi, ce qui contribue à leur autonomie et à leur indépendance.

Concrètement, la Coopérative de la Vallée Bras-du-Nord n'est pas un organisme à but non lucratif, mais une organisation collective privée : les revenus générés sont réinvestis dans l'entreprise pour consolider les emplois des membres travailleurs, pour développer des infrastructures, telles que les chalets d'accueil, et en assurer la pérennité.
Concrètement, la Coopérative de la Vallée Brasdu-Nord n'est pas un organisme à but non lucratif, mais une organisation collective privée : les revenus générés sont réinvestis dans l'entreprise pour consolider les emplois des membres travailleurs, pour développer des infrastructures, telles que les chalets d'accueil, et en assurer la pérennité ${ }^{3}$.

La trentaine d'initiatives d'envergure réalisées au cours des 6 dernières années, et qui concernent des actions écotouristiques entreprises auprès des populations résidantes, des entrepreneurs forestiers et des partenaires économiques ${ }^{\mathrm{f}}$, permet également de prendre la mesure de la démarche de développement durable au sein de la Coopérative. La tenue d'un forum d'experts sur les différents enjeux concernant le territoire et les activités de la vallée, ou encore la signature d'entente de droits de passage entre promoteurs écotouristiques et propriétaires fonciers, en sont des exemples. La Coopérative a été par ailleurs récipiendaire de plus d'une dizaine de prix au niveau national et régional. Elle est citée en exemple partout au Québec et maintenant à l'international comme « un modèle extraordinaire de tourisme durable intégrant à la fois les jeunes, la population locale et les intervenants locaux $»^{\mathrm{g}}$.

La mise en place de l'évènement La Fête champêtre est un autre exemple de la volonté d'intégration des questions liées à l'exploitation écotouristique des territoires. Cette fête citoyenne a réuni à deux reprises, sur le thème du développement durable et de l'écoresponsabilité, des intervenants des secteurs de l'agroalimentaire, du récréotourisme, de l'environnement et de la culture. Cette démarche emblématique illustre le souci de valoriser une approche holistique, intégrée, qui fait à contrario largement défaut dans la plupart des projets écotouristiques privés (Tourisme Québec, 2002; CQRHT, 1996) :

L'approche privilégiée pour le développement écotouristique de ce territoire intègre les quatre dimensions du développement soit : les dimensions culturelle, économique, sociale et environnementale. La démarche touristique, dont l'aspect central est la préservation des paysages naturels, s'inscrit comme une action transversale qui participe à l'affirmation d'une nouvelle culture territoriale ${ }^{4}$. 
L'inscription du projet dans un objectif de DD apparaît ainsi dans son mandat initial, ses plans stratégiques successifs, sa structure administrative, ses réalisations concrètes, et les valeurs sur lesquelles la direction communique. C'est également la reconnaissance du milieu professionnel et les résultats objectifs qui témoignent de la réussite du projet et du soin apporté à une véritable structuration de la démarche de durabilité. En considérant ces résultats, une analyse transversale des démarches de DD, menées par l'ensemble des promoteurs écotouristiques au Québec au cours des dernières années, ne manquerait certainement pas de souligner le caractère exemplaire du projet de la Vallée Bras-du-Nord. Le choix de ce territoire comme étude de cas concernant l'ingénierie d'opérationnalisation de la durabilité écotouristique semble donc se justifier. Il apparaît d'autant plus pertinent que la démarche de développement durable de la Coopérative s'est matérialisée de manière encore plus patente dans le cadre du projet Tourisme, paysage et coopération (2007-2011).

\subsection{Les objectifs de développement durable promus}

En 2007, la Coopérative a obtenu une subvention de $136000 \$$ du Fonds d'action québécois pour le développement durable (FAQDD), afin de réaliser un plan de développement intitulé Tourisme, paysage et coopération ${ }^{\mathrm{h}}$. Ce plan de développement repose sur des objectifs génériques et spécifiques. Les premiers visent à mettre en œuvre les seize principes directeurs de la Loi sur le développement durable du Québec (2006) ${ }^{\mathrm{i}}$, les seconds, à réaliser de manière plus précise un certain nombre d'opérations ciblées, présentées ci-après.

Le projet soumis au FAQDD impliquait que la Coopérative s'efforce de mettre en place un plan de développement s'inscrivant dans le cadre de la Loi sur le développement durable du Québec, adoptée en avril 2006. Cette loi établit seize principes de DD, qui s'inspirent de la Déclaration de Rio de 1992. Bien qu'elle ne s'applique pas encore aux organisations hors de l'appareil gouvernemental, les membres de la Table d'accompagnement-conseil des entreprises du secteur privé (TACEP) ont travaillé à l'élaboration d'une stratégie de sensibilisation des entreprises par rapport au développement durable, qui inclut la mise sur pied du Projet BNQ 21000'. La Coopérative s'est inspirée de cette démarche pour établir son propre plan d'action de développement durable. L'objectif était notamment d'inclure dans le projet tous les groupes d'individus qui se partagent ce territoire : résidents, forestiers, promoteurs touristiques, amateurs de plein air, villégiateurs ${ }^{5}$. Le plan de DD est un plan stratégique, centré sur trois méta-objectifs :

1. la protection du territoire et des paysages;

2. le développement et la diversification économiques;

3. la coopération et la participation des différentes parties prenantes.

Ces objectifs correspondent aux trois dimensions du DD : environnementale, économique et sociale.

Le plan de DD de la Vallée Bras-du-Nord vise également à rencontrer certains objectifs spécifiques, inspirés des principes de la Loi 118, mais adaptés aux réalités territoriales, aux pratiques écotouristiques, et aux besoins prioritaires relevés par les gestionnaires du site :

- «Protéger le territoire (l'aspect champêtre, les paysages, le patrimoine bâti, les peuplements forestiers, les rives de la rivière et de ses affluents, dont la chute Delaney);

- éviter que le développement touristique soit une source de nuisance à l'environnement et à la communauté locale;

- modifier les comportements des amateurs de plein air (bonnes pratiques en écotourisme "sans trace", respect des propriétés privées, etc.);

- susciter la coopération entre les différents acteurs afin de maintenir l'équilibre entre le développement touristique, l'exploitation forestière, la villégiature, l'environnement et la population locale;

- élargir la participation active de la population, des entreprises touristiques, des travailleurs forestiers, des visiteurs, des intervenants (municipaux et gouvernementaux) à la mission de la Coopérative de solidarité;

- contribuer à diversifier l'économie d'une communauté agroforestière dans une démarche de développement durable ».

(Source : Vallée Bras-du-Nord, 2007 : 5) 


\subsection{La méthode d'opérationnalisation du plan d'action de développement durable}

Pour réaliser leur plan d'action de développement écotouristique durable, les gestionnaires de la Coopérative ont mis en place une planification stratégique qu'ils ont ainsi définie :

Les promoteurs du projet travailleront d'abord à évaluer de façon précise le potentiel écotouristique de la Vallée, en concertant tous les partenaires locaux. Ils élaboreront ensuite des trousses d'action selon trois thèmes: la construction de villégiature verte (chalets), l'adoption de pratiques forestières assurant la protection des paysages et la sensibilisation au tourisme responsable. Les entreprises touristiques et leurs clients seront notamment initiés aux pratiques dites d'écotourisme « sans trace » (réduction de l'impact environnemental des activités de plein air). Par ailleurs, pour favoriser une gestion durable de la Vallée, l'équipe travaillera avec des experts et des acteurs municipaux pour proposer un cadre normatif original et adapté à la réalité d'un parc écotouristique habité. Finalement, la Coopérative évaluera son expérience et en partagera les résultats avec les réseaux nationaux d'acteurs impliqués en environnement, écotourisme et coopération ${ }^{5}$.

Les objectifs et la séquence étant posés, les gestionnaires de la Coopérative ont alors produit un cadre d'intervention, ciblant les opérations à mener pour mettre en œuvre chacun des seize principes de la loi, et, au travers d'eux, réaliser également les objectifs spécifiques mentionnés plus haut. Ce cadre d'intervention est présenté dans le tableau de l'annexe 1 du présent article.

\section{DE LA PAROLE AUX ACTES : LA MISE EN EUVRE D'UNE DURABILITÉ ÉCOTOURISTIQUE}

L'objectif de la présente recherche est d'évaluer l'efficience - le rapport entre les résultats obtenus et les ressources utilisées - d'outils et de méthodes employés pour mettre en œuvre des objectifs de développement durable en contexte écotouristique. À ce titre, le plan stratégique élaboré par les gestionnaires de la Vallée Bras-du-Nord, à partir des principes de la Loi 118, peut servir de grille d'analyse. Cependant, parce que le cadre de mise en œuvre des objectifs de développement durable est l'écotourisme, l'hypothèse que nous formulons ici est que les critères de la durabilité écotouristique, plus précis que les principes génériques du $\mathrm{DD}$, pourraient être avantageusement utilisés. Ainsi, les données ont été évaluées au moyen d'une grille de 35 critères de la durabilité écotouristique, dont seuls ont été conservés les critères correspondant précisément aux objectifs visés par le plan d'action de la Coopérative.

Cette hypothèse se fonde sur deux éléments. Le premier est que le caractère générique et transversal des principes issus de la loi pourrait être un frein à leur mise en œuvre pratique, compte tenu de leur nécessaire adaptation aux spécificités de l'écotourisme. Le sens donné à tel ou tel principe a ainsi pu varier entre l'approche normative du FAQDD et celle, davantage praticienne, des gestionnaires. Le second est qu'une précédente recherche ${ }^{6}$ a précisément relevé
35 critères de la durabilité écotouristique, soit des critères contributifs aux dimensions économique, environnementale et sociale du développement durable des territoires soumis à l'exploitation écotouristique. C'est à partir de cette dernière grille que l'évaluation a été conduite. Cette évaluation porte sur l'efficience des outils, les étapes du cycle de vie du projet au cours desquelles ces outils ont été employés et leur capacité d'adaptation aux spécificités locales.

2.1. Analyse de l'efficience entre critères de durabilité écotouristique et mise en ouvre du plan d'action de développement durable de la Coopérative

À partir de la revue de littérature praticienne, il a pu être précisé, d'une part, quel outil technique a été sollicité pour atteindre les objectifs ciblés et, d'autre part, quels résultats ont été obtenus. L'étape de la gestion du projet, lors de laquelle les outils ont été employés, est également précisée, afin d'estimer la cohérence dans leur séquence d'utilisation.

Le tableau 1 présente les résultats de recherche. En s'y référant, le relevé des outils sollicités pour atteindre les objectifs de DD ciblés permet de faire certaines observations d'intérêt. La première (1) a trait aux critères et objectifs visés; la deuxième (2), à la nature des outils utilisés et priorisés; la troisième (3), à leur efficience respective 


\begin{tabular}{|c|c|c|c|c|c|}
\hline 这 & 㓙 & గ્ર & तु $\infty$ & & న్ ळ \\
\hline 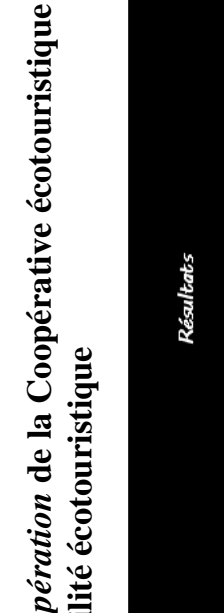 & 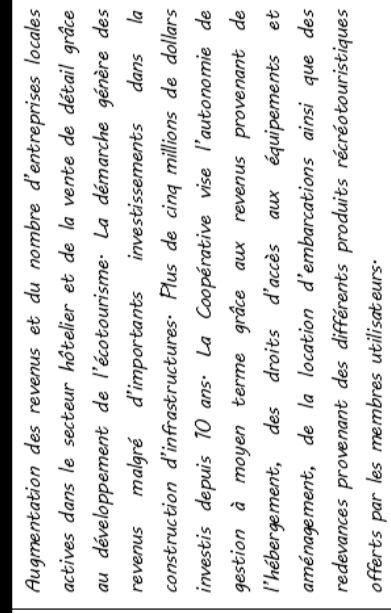 & 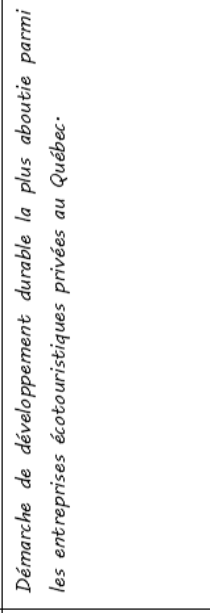 & 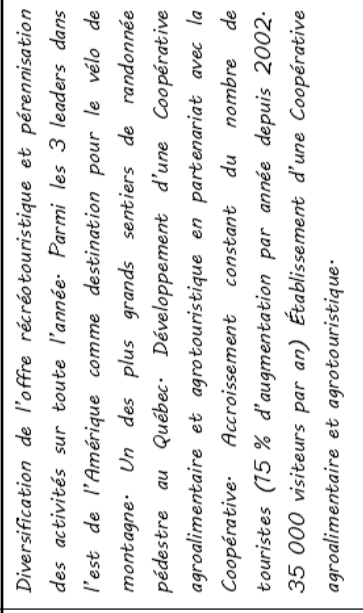 & 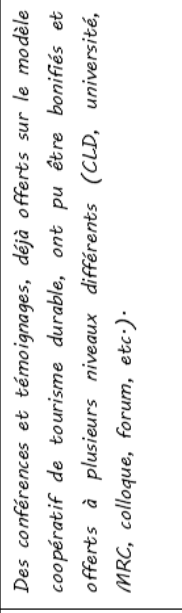 & 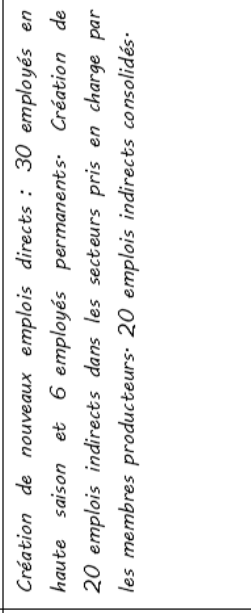 \\
\hline 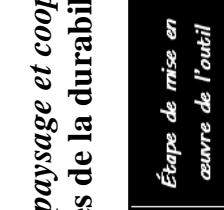 & 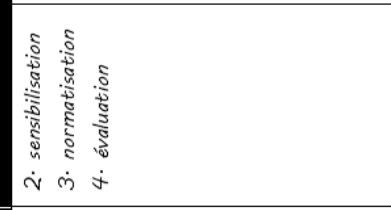 & 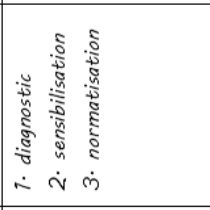 & 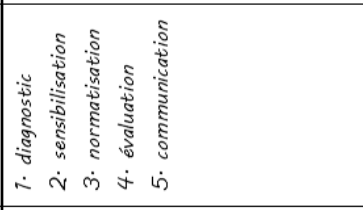 & & 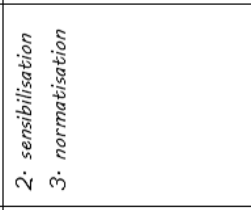 \\
\hline 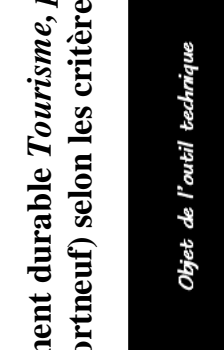 & 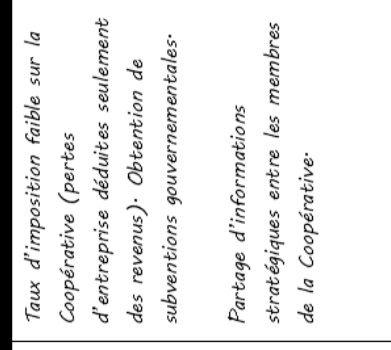 & 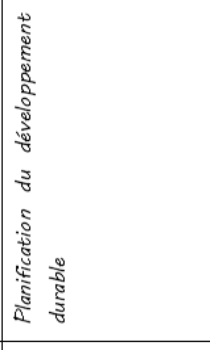 & 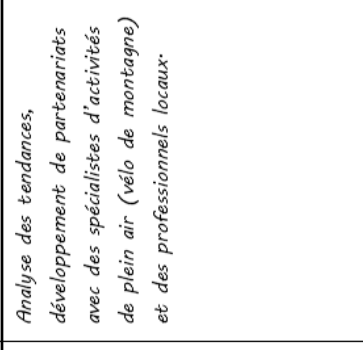 & 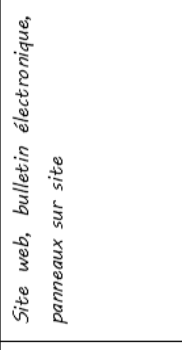 & 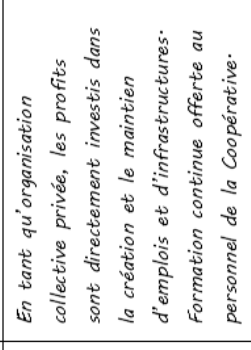 \\
\hline 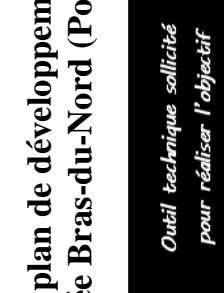 & 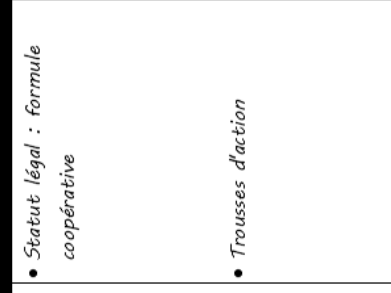 & 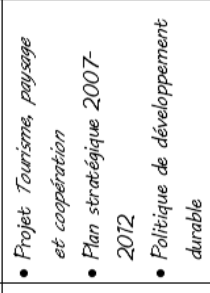 & 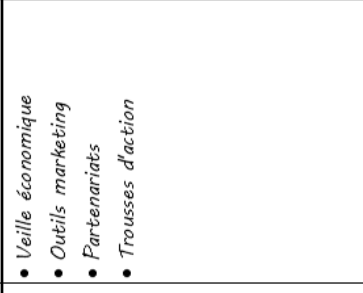 & 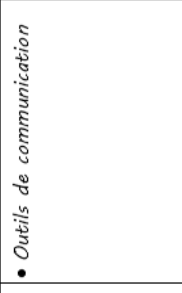 & 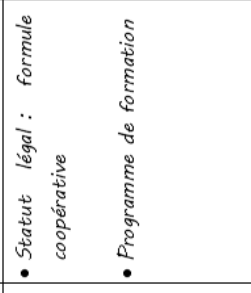 \\
\hline 尊 & 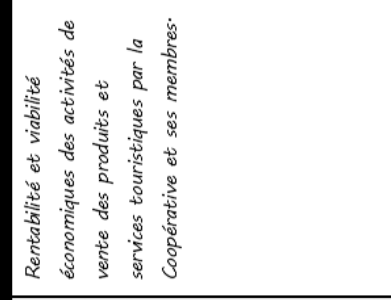 & 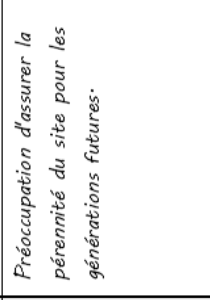 & 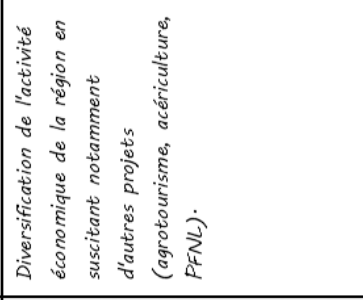 & 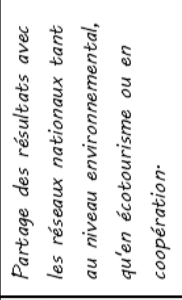 & 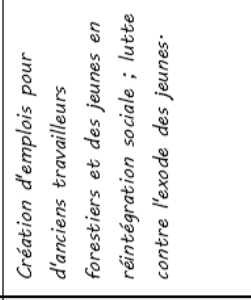 \\
\hline 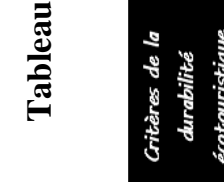 & 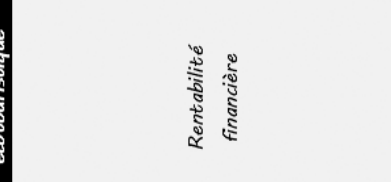 & 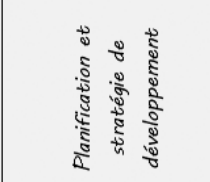 & 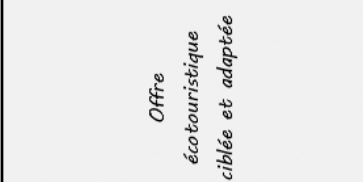 & 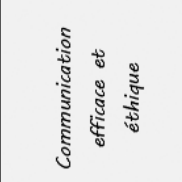 & 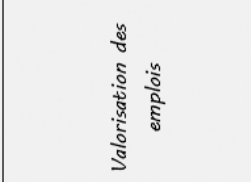 \\
\hline
\end{tabular}




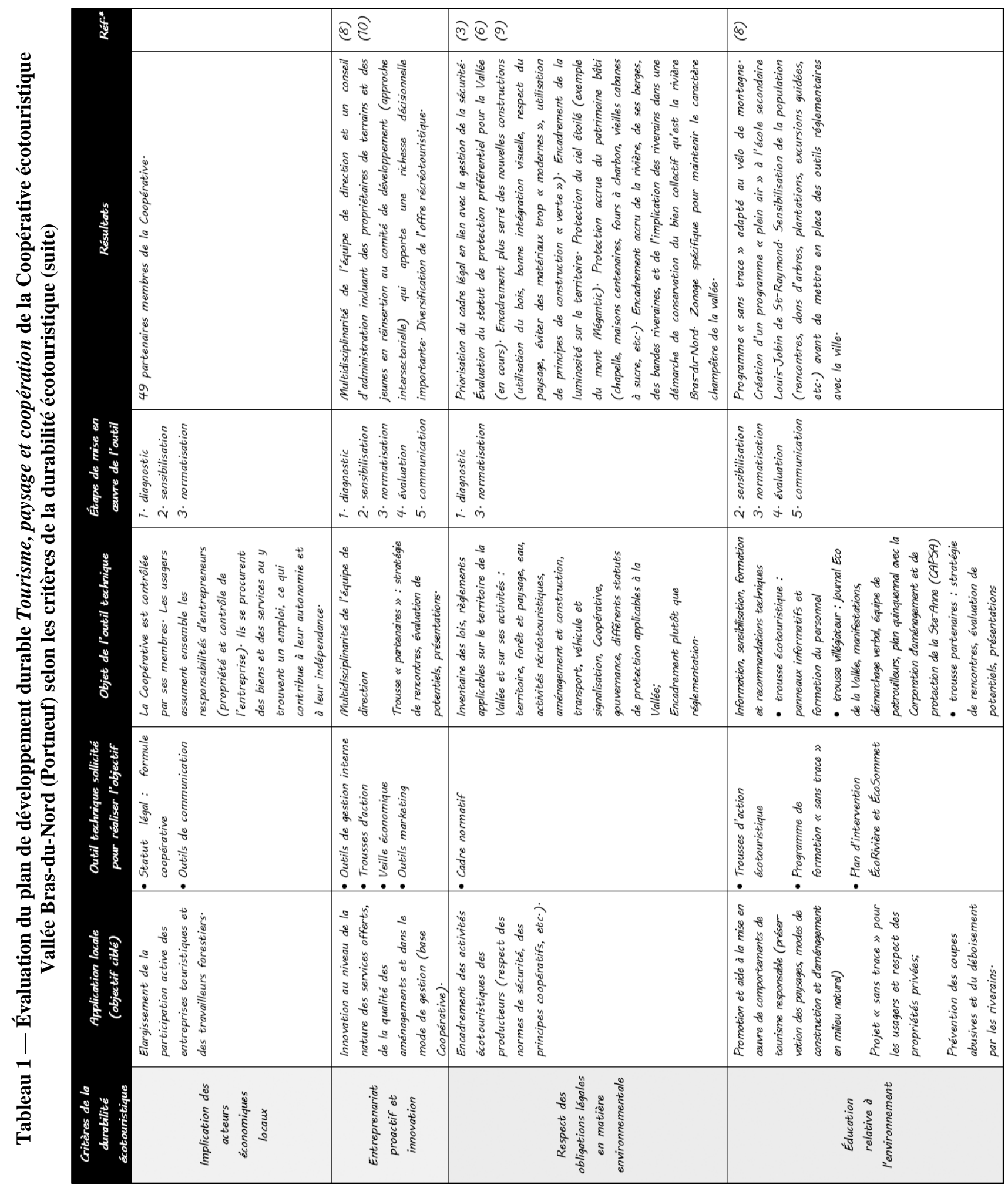




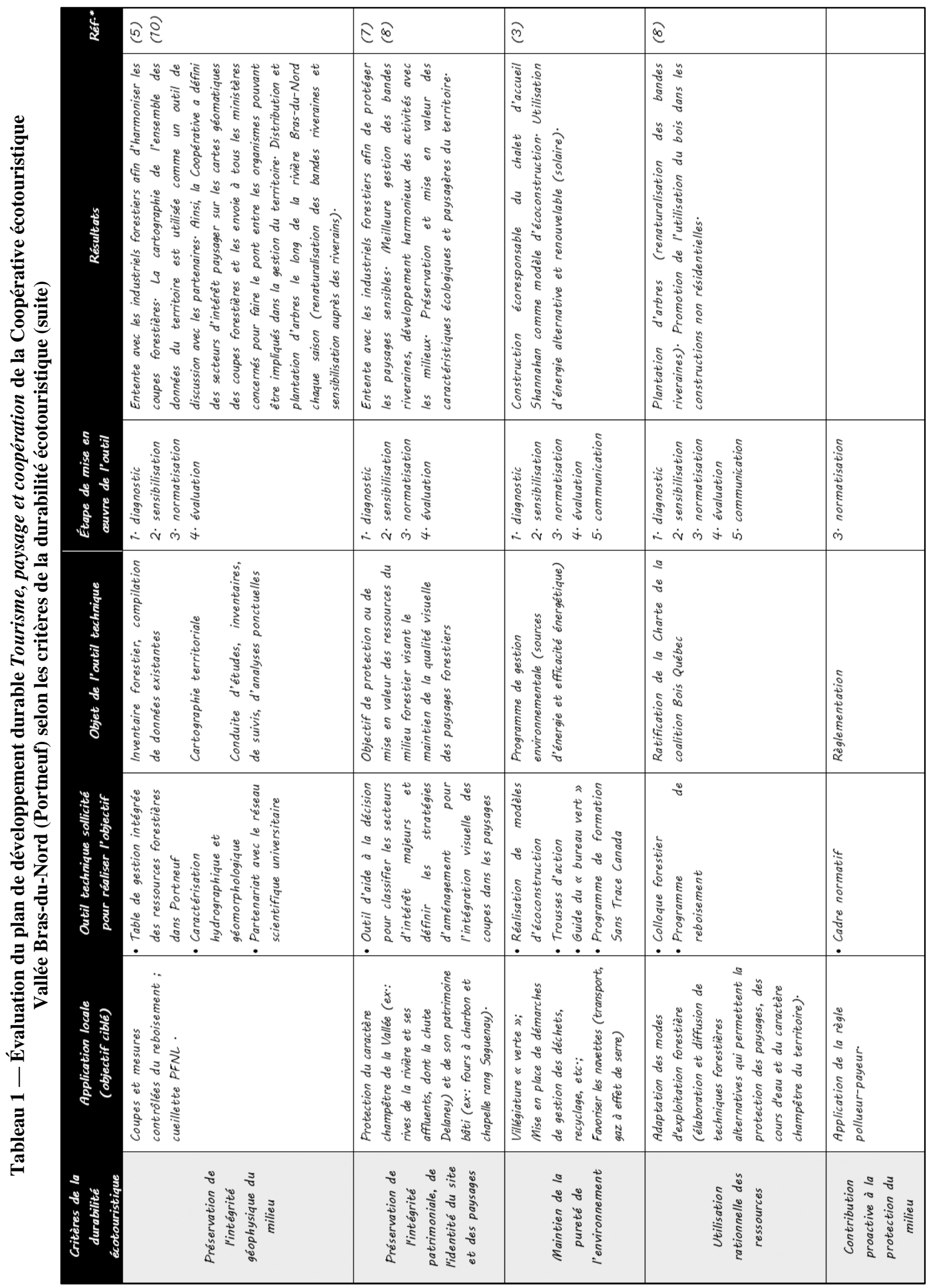




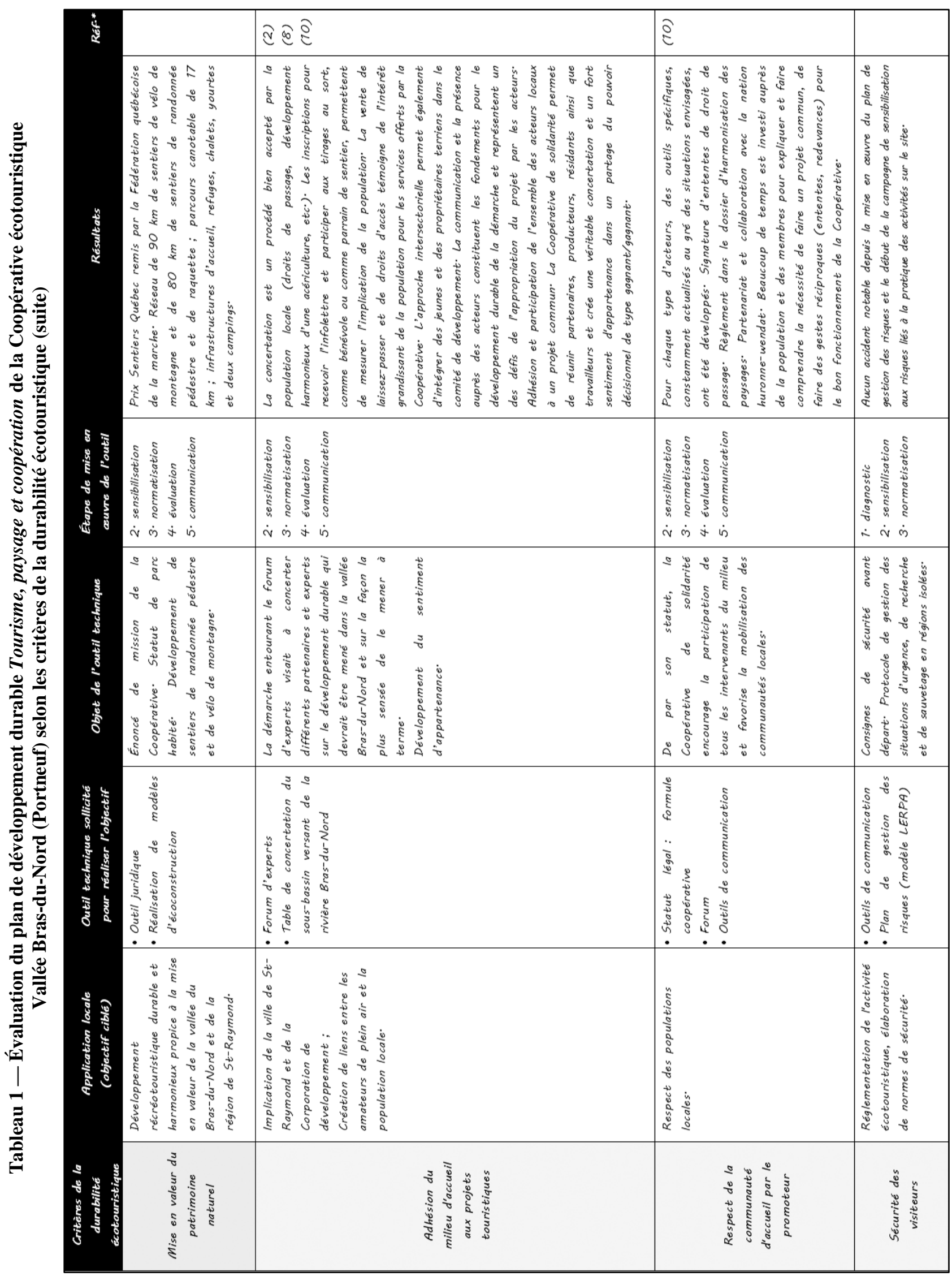




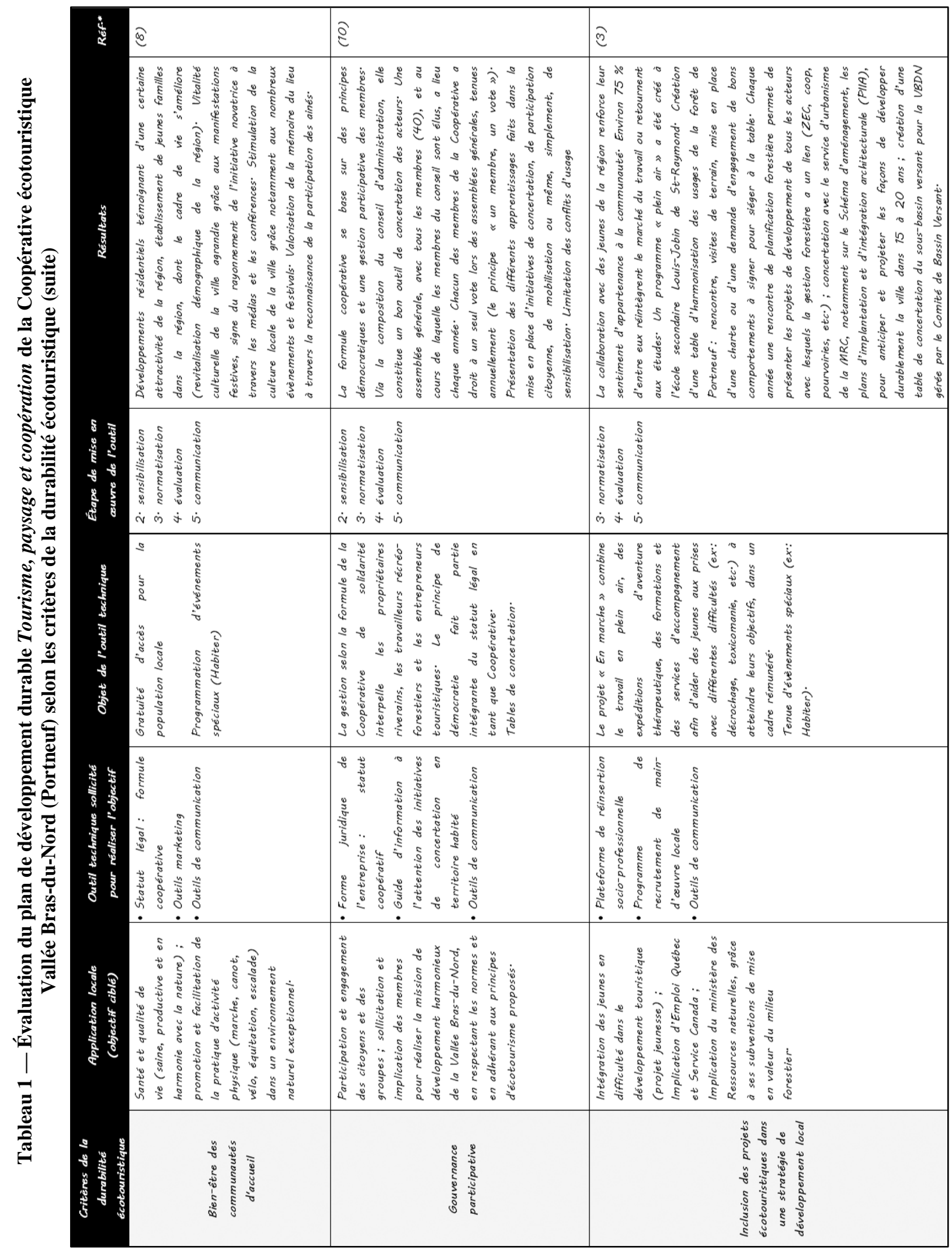




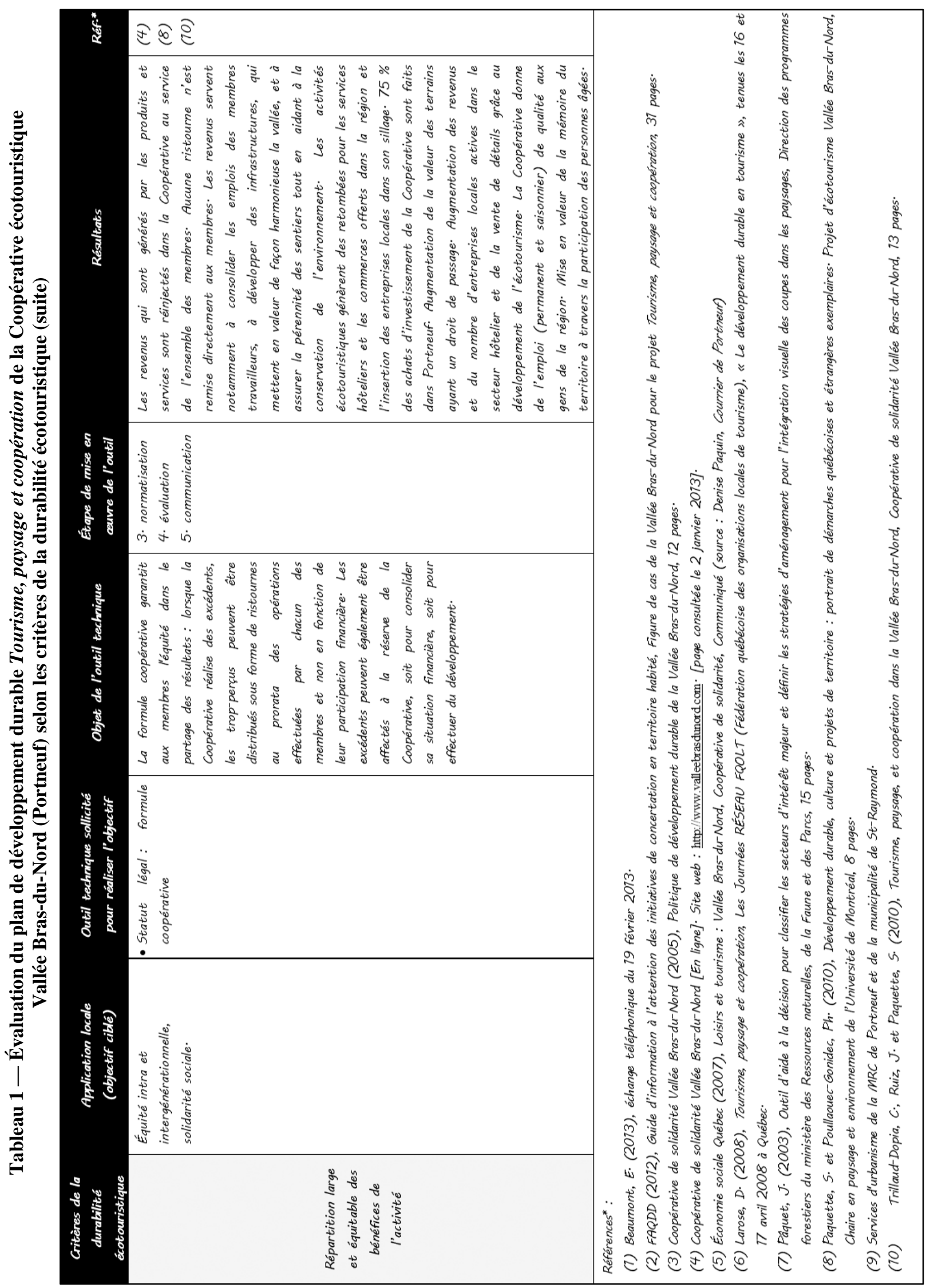


1. Le projet Tourisme, paysage et coopération aborde 23 des 35 critères génériques $(66 \%)$ de la durabilité écotouristique tels que retenus dans notre grille d'analyse (Tranquard et Gagnon, 2012). Les critères non considérés concernent essentiellement l'encadrement et le bien-être des visiteurs, l'efficacité marketing et le contrôle de la qualité et, de manière générale, l'efficacité économique.

Parmi les critères mis en œuvre (colonne 1), notons une bonne intégration de toutes les dimensions du DD. Par ordre d'importance, ces critères portent sur la dimension environnementale $(n=9)$, sociale $(n=7)$ et économique $(n=7)$. La légère surreprésentation des critères environnementaux, mise en perspective avec les bons résultats généraux de la Coopérative, pourrait contribuer à valoriser l'idée que la gestion des ressources naturelles est prioritaire et centrale dans le développement durable de l'écotourisme.

2. La nature des outils révèle quant à elle que ceuxci sont diversifiés et peuvent être catégorisés (colonnes 3 et 4). À partir des données obtenues, les outils employés par la Coopérative sont de six natures différentes :

- outils d'analyse scientifique (ex.: protocoles, méthodes d'inventaire, guides scientifiques);

- outils juridiques (ex.: encadrement, règlementation);

- outils marketing (ex. : partenariats, mise en marché, veille);

- outils de communication et de diffusion (ex. : conférences, publications);

- outils didactiques et de formation (ex. : guides, programmes d'action de terrain);

- outils de concertation et de négociation (ex. : forums, tables de concertation et de gestion intégrée).

Parmi ces six types d'outils techniques, les plus sollicités sont les outils de formation et de concertation. C'est par exemple le cas pour favoriser le critère Adhésion du milieu d'accueil aux projets touristiques. Mais c'est surtout l'outil juridique qui apparaît de manière transversale, en ce que le choix du statut coopératif est davantage priorisé dans la démarche de développement durable: «Selon nous, notre formule coopérative s'avère être une voie d'avenir innovatrice tant au niveau de l'intégration du développement durable dans la mise en valeur des territoires que dans le développement du tourisme responsable et de la notion de paysage comme outil de développement économique d'une région (ou comme levier économique) $»^{k}$. Les bénéfices liés à la formule de la coopérative de solidarité apparaissent tant au niveau de la capacité de participation des populations qu'à la conduite de négociations, au partage des bénéfices et à la construction d'un sentiment d'appartenance, notamment (Langlois, 2006).

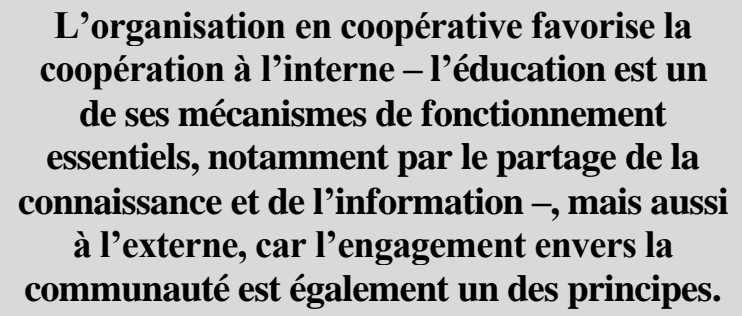

L'organisation en coopérative favorise la coopération à l'interne - l'éducation est un de ses mécanismes de fonctionnement essentiels, notamment par le partage de la connaissance et de l'information -, mais aussi à l'externe, car l'engagement envers la communauté est également un des principes. Le modèle s'articule autour de la notion de «bonne gouvernance ${ }^{7}$ » puisque la Coopérative est avant tout une entreprise collective, au sens premier du terme. La gouvernance représente « un processus dynamique de coordination de l'action collective dans laquelle la mobilisation des acteurs et la formation en réseaux constituent une communauté plus riche en ressources, plus expérimentée et plus flexible ». La participation directe et constante de ses membres à sa gestion constitue notamment un des principes qui fondent cette structure originale. En termes d'ingénierie touristique, l'origine de ce processus repose sur le recours à l'outil juridique comme support au choix de la structure organisationnelle.

Il doit également être souligné qu'un outil privilégié par les gestionnaires de la Coopérative pour favoriser le développement durable est la trousse d'actions. C'est par exemple le cas pour favoriser l'atteinte des objectifs économiques ou encore l'éducation relative à l'environnement. Concrètement, il s'agit d'un outil composite intégrant des outils d'information, de sensibi- 
lisation et des modes d'emploi techniques. À titre d'exemple, la trousse d'actions écotouristiques cible la clientèle, les visiteurs du site. Cet outil comprend un programme de formation aux bonnes pratiques en écotourisme "Sans trace », ainsi que des journées de sensibilisation aux caractéristiques du milieu naturel et habité de la vallée dans le cadre d'activités appelées ÉcoRivière et ÉcoSommet. Des ateliers pratiques ponctuent ces activités.

3. La question de l'efficience des outils est quant à elle plus problématique. L'efficience se mesure par le ratio entre les résultats obtenus et les ressources utilisées pour parvenir à ces résultats. Le résultat visé est ici le développement durable. En l'espèce, l'évaluation de l'efficience des outils techniques utilisés pour favoriser la durabilité de la Vallée Bras-du-Nord repose sur la question de savoir si la mise en œuvre des outils a été optimale ou encore si d'autres outils auraient pu être sollicités avec plus de succès.

Une impression d'ensemble largement positive semble se dégager du projet de développement durable de la Coopérative. Les résultats indiqués dans la colonne 6 du tableau 1 , et obtenus de la Coopérative elle-même, et les références listées dans la dernière colonne illustrent la cohérence et l'ampleur des démarches entreprises et l'omniprésence de l'objectif de durabilité dans chacun des sous-projets pilotés par les gestionnaires ${ }^{3,4,9}$. Un moyen plus objectif d'évaluer l'efficience est cependant d'analyser les actions restant à conduire pour tendre vers une démarche exhaustive de DD. Parmi ces actions, notons :

- l'atteinte de la rentabilité financière et l'autofinancement;

- le maintien de la croissance : positionnement de la vallée comme première destination de vélo de montagne dans l'est de l'Amérique du Nord et attrait d'une clientèle internationale;

- la conciliation des usages multiples du territoire et notamment de l'écotourisme avec les activités préexistantes au projet (élevage, quad, chasse, etc.);

- le repli de certains partenaires lié à des frustrations qui restent non évoquées;
- l'influence de partenaires au fort pouvoir économique et politique qui peut freiner des décisions auparavant concertées;

- l'arrimage entre l'échéancier de production de projets et le temps long de la concertation entre de nombreux partenaires économiques et politiques;

- l'évaluation de la pertinence et de l'impact de l'obtention d'un statut de parc régional.

Ces actions complémentaires découlent de certains critères de la durabilité écotouristique qui n'ont pas été abordés jusqu'à présent par la Coopérative, comme le contrôle de l'efficacité économique par exemple. L'atteinte de ces objectifs nécessiterait par ailleurs de recourir à des outils nouveaux. Si en effet des outils marketing, juridiques et de concertation sont de nature à faciliter l'atteinte de certains objectifs cités plus haut, ce sont surtout des outils de gestion qui semblent être requis. Ces outils sont par nature plus appropriés pour faciliter le contrôle et le suivi des états financiers, la mise en place d'une démarche de qualité transversale, la gestion des interactions visiteurs/biodiversité, ou encore la coordination des agents économiques (maillage d'entreprises, réseautage économique à l'extérieur de la communauté), démarches qui correspondent spécifiquement aux objectifs complémentaires. Ce constat tendrait selon nous à indiquer que d'autres outils pourraient être utilisés pour potentialiser davantage le développement durable selon une approche holistique.

\subsection{Analyse du cycle de vie du projet écotouristique durable}

Le plan de travail initial, pour réaliser le projet de développement durable Territoire, paysage et coopération, a été établi en quatre étapes. Ces étapes consistaient en :

(1) une mise en commun des connaissances et une concertation des partenaires;

(2) des connaissances, une sensibilisation, des trousses d'action;

(3) un encadrement normatif;

(4) une évaluation et une promotion de l'initiative.

Il s'agit maintenant d'évaluer s'il existe une certaine cohérence dans cette séquence, où chaque étape permettrait de conduire la suivante de 
manière efficiente. Il s'agit également d'estimer si, pris dans son ensemble, ce cycle d'actions favorise la mise en œuvre d'un développement durable.

La première étape est une étape de diagnostic, d'inventaire. Cet inventaire porte sur le potentiel écotouristique du territoire et ses limites environnementales, le cadre juridique applicable et les contraintes de réalisation du projet. $\mathrm{La}$ deuxième étape correspond à la diffusion et au partage des informations colligées précédemment. Plus encore, elle a permis de sensibiliser et de fédérer l'ensemble des parties prenantes aux objectifs collectifs, de fixer des balises et d'établir certains objectifs ponctuels. Le but de la troisième étape était d'entériner les objectifs, de les traduire en obligations légales et contractuelles, afin que le plan de développement durable devienne le cadre normatif de toutes activités sur le territoire. Enfin, la dernière étape était double : une évaluation de la mise en œuvre du projet a été conduite, puis les gestionnaires se sont investis dans la diffusion et la communication des résultats de la démarche.

De prime abord, l'enchaînement des étapes paraît cohérent, en ce qu'il traduit une progression dans la mise en place d'un plan d'action. La deuxième étape n'est possible qu'à la suite de la première qui elle-même conditionne et alimente la troisième, et ainsi de suite. Cette progression est reprise dans ses grandes lignes par l'Organisation mondiale du tourisme $^{10}$, dans ses documents techniques ainsi que par certains spécialistes de la gestion de projets touristiques. Ainsi la Coopérative a bel et bien mis en œuvre un diagnostic des ressources et de leur environnement. Désignée par diverses appellations (état des lieux, tourism resource audit, analyses des composantes physiques et spatiales), cette étape est incontournable et prioritaire en raison de son caractère générique $^{11}$. La phase d'information des parties prenantes, qui suit normalement le diagnostic du territoire, est elle aussi documentée.

En revanche, l'analyse des marchés ou Market Research vient généralement compléter l'étape d'évaluation du potentiel du territoire. Cette étape n'est pas clairement identifiée ici. De la même manière, une étape importante est requise avant l'instauration d'un cadre normatif paramétrant les objectifs, laquelle ne transparaît pas expressément dans la séquence opérée par la Coopérative. Cette étape est celle du projet détaillé des étapes et manifestations concrètes du plan d'action, Product Development Planning (GNWT, SD) ou encore Tourism Development Action Steps ${ }^{11}$. Elle consiste en la formulation précise de sousobjectifs, incluant un échéancier, un programme d'attribution et de gestion des ressources, des mécanismes de contrôle, etc. La juxtaposition d'objectifs ponctuels ne peut en effet se substituer à une planification globale priorisant et mettant en perspective ses objectifs ciblés. Ce constat tend à corroborer l'observation faite plus tôt, à l'effet qu'une méthodologie exhaustive de gestion de projets est indispensable à la conduite de projets écotouristiques visant le développement durable.

Dans le cas étudié, il apparaît que le passage entre le diagnostic et l'implantation de directives spécifiques est relativement rapide. Une raison serait qu'un plan d'action précis, validé à chaque stade par les parties prenantes, semble faire défaut. La normatisation des objectifs et contraintes ne semble pas totalement reposer sur les mécanismes de concertation mis en place au cours du projet.

L'analyse des dernières étapes peut également susciter certains commentaires. L'évaluation de l'atteinte des objectifs y apparaît comme un processus unique, ponctuel. Même si celle-ci porte uniquement sur les objectifs précis du projet Territoire, paysage et coopération, la lettre et l'esprit d'une démarche de développement durable semblent commander que l'évaluation se fasse sur une base plus fréquente pour ne pas dire systématique et itérative. Un cycle plus court d'évaluation favoriserait le partage d'informations entre les parties prenantes, les ajustements conjoncturels, les réorientations structurelles ${ }^{11}$, le suivi de la satisfaction de la clientèle ou encore l'analyse de la rentabilite ${ }^{12,13}$.

La logique d'intervention, qui a été envisagée dans le plan de développement ou plan d'action, gagnerait par ailleurs à être confrontée à sa mise en œuvre réelle. Dans les faits, le relevé des étapes au cours desquelles les outils ont réellement été employés permet de souligner que l'étape 2 de sensibilisation apparaît comme un fil conducteur, en ce sens qu'elle a été intégrée à la quasi-totalité des démarches de DD. L'étape de diagnostic a surtout porté sur les composantes environne- 
mentales du site sans y intégrer l'étude du tissu socio-économique. Les critères relevant de la dimension environnementale de la durabilité écotouristique sont par ailleurs ceux qui ont le plus donné lieu à une mise en œuvre exhaustive des étapes du plan de développement.

Reste alors à évaluer si l'ingénierie de la durabilité écotouristique, dont les modalités se dessinent progressivement dans le cadre du projet Territoire, paysage et coopération, a vocation à devenir un modèle générique pour les gestionnaires de projets écotouristiques.

\subsection{Prise en compte des critères territoriaux de développement durable}

Le dernier point présenté ici porte sur la question de savoir si l'identification et la prise en compte de particularités ou spécificités territoriales sont nécessaires à la mise en œuvre d'une durabilité écotouristique, et influent sur l'usage des outils utilisés à cette fin. Les particularités de nature à «territorialiser» un plan d'action de DD sont par exemple une variété d'usages sur un même site, un historique d'exploitation des ressources forestières ou de collaboration avec le milieu municipal, le statut coopératif de l'organisme mettant en valeur le territoire, etc. En l'espèce, est-ce que la démarche mise en œuvre dans le cadre du projet Territoire, paysage et coopération est transposable en l'état à tout territoire, ou bien inclut-elle des spécificités locales propres à la Vallée Bras-duNord, qui la rendent unique? De quels facteurs locaux d'autres promoteurs qui souhaiteraient emprunter la même méthodologie devraient-ils particulièrement tenir compte s'ils opèrent dans un contexte socio-économique et environnemental similaire?

Il apparaît tout d'abord que le territoire écotouristique de la Vallée Bras-du-Nord est porteur de particularismes. Un de ceux-ci réside dans son zonage. Ce territoire, habité depuis près d'un siècle et demi, regroupe des terrains privés, des terres du domaine de l'État et certains zonages spécifiques, comme les ZEC, les refuges biologiques ou les écosystèmes forestiers exceptionnels. Cet état de fait réside notamment dans la superficie particulièrement importante du territoire écotouristique $\left(50 \mathrm{~km}^{2}\right)$. Une partie de celui-ci est par ailleurs un territoire de la Nation huronne-wendat et, à ce titre, n'est pas soumise aux mêmes règles de gestion que le reste du territoire, ce qui en rend la gouvernance plus problématique.

Autre particularisme local, le projet en est un éminemment collectif. Cette situation peut être facteur de contraintes additionnelles en termes de concertation ou de reddition de comptes ou, au contraire, être un atout lorsqu'on pense davantage aux modalités de financement. La Coopérative compte 49 membres incluant la Nation huronnewendat et 20 partenaires stratégiques (AEQ, ministère des Ressources naturelles, Fédération québécoise de la marche, etc.), et bénéficie notamment de l'implication de diverses instances gouvernementales. Ce réseautage important s'étend à des agences spécialisées (Sans Trace Canada par exemple), qui soutiennent en même temps qu'elles confèrent une crédibilité à certaines démarches de développement durable. Ce réseautage se traduit également dans le support obtenu à la diffusion d'informations, de la part à la fois de médias locaux mais aussi spécialisés.

Une autre particularité tient à la multidisciplinarité de l'équipe de direction. Cette multidisciplinarité, pas courante dans le secteur écotouristique (CQRHT, 2005 : 6), semble bénéficier au projet écotouristique en plusieurs occasions. La polyvalence des gestionnaires les conduit par exemple à s'investir avec succès dans un très grand nombre de forums d'échanges (table de concertation, organisme de bassin versant, etc.), de projets scientifiques et de communication, qui servent et alimentent l'objectif de développement durable. Cette même multidisciplinarité nourrit une forme de leadership qui semble rassurer les partenaires de la Coopérative en même temps qu'elle est un gage d'efficacité entrepreneuriale et d'ouverture. Cette ouverture se traduit par exemple par l'intérêt particulier porté à la problématique de sauvegarde des paysages, composante du DD peu abordée, surtout dans le secteur privé.

Une dernière particularité est la présence, au sein de la démarche écotouristique, d'un projet fédérateur et emblématique, le projet «En marche». Ce projet de réinsertion socioprofessionnelle, basé sur des expéditions d'aventure thérapeutique et de formation sur le travail récréoforestier, est particulièrement original et efficace. En 10 ans, 
120 jeunes ont œuvré à l'aménagement de plus de $120 \mathrm{~km}$ de sentiers, et près de $75 \%$ d'entre eux ont par la suite réintégré le marché du travail ou sont retournés aux études. Ce projet mérite d'être souligné en raison du fait qu'il opère une sorte de synthèse entre les objectifs de développement durable: la préservation de l'environnement comme condition, l'économie comme moyen et le social comme finalité.

Parce qu'elles sont propres au contexte territorial de la Vallée Bras-du-Nord, ces particularités peuvent représenter tour à tour une contrainte ou un élément facilitateur pour l'implantation d'une démarche de développement durable. Ainsi la multidisciplinarité des gestionnaires apparaît clairement comme facilitant l'utilisation d'outils d'ingénierie variés et adaptés et, au final, favorise la démarche de $\mathrm{DD}^{14}$. A contrario, la multiplicité des parties prenantes et la variété de statuts des

\section{CONCLUSION}

Les enseignements à tirer de cette étude de cas alimentent directement nos questions initiales de recherche. La mise en œuvre du plan de développement durable du territoire de la Vallée Bras-du-Nord souligne en effet qu'il existe un lien d'efficience entre l'atteinte des objectifs de développement durable et les outils utilisés dans le cadre d'un projet de développement écotouristique, et notamment la pertinence de certains outils sites d'activité obligent à recourir à des outils spécialisés - cadre normatif par exemple. Les spécificités locales influencent ainsi le contenu d'un plan d'action de DD, puisque

[L]e développement durable se traduit, au niveau des territoires, par des enjeux différents, mais également des rapports différents entre les acteurs et la démarche elle-même. Les enjeux territorialisés de développement durable se rapportent à des réalités, un niveau de maitrise à un moment donné, une volonté et une capacité de mobilisation qui définissent un contexte local. (Ponrouch, 2008 : 111).

Leur prise en compte, leur caractérisation et leur reconnaissance font partie intégrante d'un mode de gestion s'inscrivant dans une démarche territoriale de développement durable.

juridiques et de communication. La prise en compte des spécificités sociales, politiques ou encore historiques du territoire y apparaît également jouer pour beaucoup dans le succès de ce projet écotouristique. Enfin, le recours aux outils d'ingénierie se réalise tout au long de la vie du projet, sans se limiter à sa phase de conception, ce qui tend à inscrire un peu plus le territoire dans une démarche de développement durable.

\section{Illustration - Vallée Bras-du-Nord (Portneuf, Québec)}

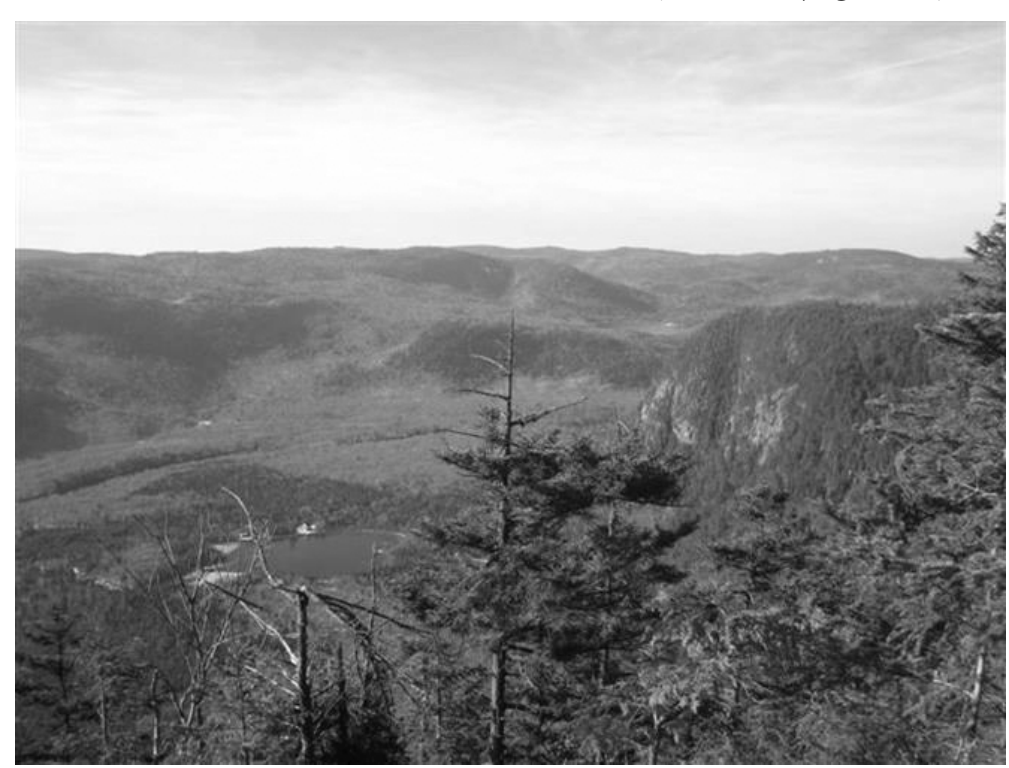

Source : Lesmarcheurs.com 


\section{BIBLIOGRAPHIE}

André, E. (1998). Méthode d'élaboration et de présentation d'un dossier de projet touristique, Paris : AFIT. 32 p.

Bell, C.; L. Ketchum et J.. Humphries (2008). Steps to Success for Rural Entrepreneurs: Starting an Ecotourism Business in Alaska, U.S. department of commerce economic development administration, s. 1. : University of Alaska Center for Economic Development. $76 \mathrm{p}$.

Carlier, B.; J.-P. Martinetti et J.-D. Gontrand (2006). La conduite de projets touristiques durables, Voiron: Territorial Éditions. 359 p.

Coopérative de solidarité Vallée Bras-du-Nord (s. d.) «Vallée Bras-du-Nord: Coop de solidarité StRaymond de Portneuf», Vallée Bras-du-Nord, <http://www.valleebrasdunord.com>, consulté le 11 janvier 2013.

Coopérative de solidarité Vallée Bras-du-Nord (2007). Guide d'information à l'attention des initiatives de concertation en territoire habité, Québec, $31 \mathrm{p}$.

Coopérative de solidarité Vallée Bras-du-Nord (2008). Politique de développement durable de la Vallée Bras-du-Nord, St-Raymond, Québec : Coopé rative de solidarité Vallée Bras-du-Nord. $12 \mathrm{p}$. <http://www.valleebrasdunord.com/images/to_politiq ue.pdf.>, consulté le 11 janvier 2013.

CQRHT - Conseil Québécois des Ressources Humaines en Tourisme (1996, 2005). Diagnostic d'ensemble des ressources humaines en tourisme, orientations et plan d'action, Rapport final, Montréal : Groupe DBSF. 210 p.

Économie sociale Québec (2007). Loisirs et tourisme: Vallée Bras-du-Nord, Coopérative de solidarité, Communiqué (source: Denise Paquin, Courrier de Portneuf)

FAQDD - Fonds d'action québécois pour le développement durable (2012). Guide d'information à l'attention des initiatives de concertation en territoire habité : Figure de cas de la Vallée Bras-duNord pour le projet Tourisme, paysage et coopération, Québec : FAQDD. 31 p.

Godfrey, K et J.Clarke (2000). The Tourism Development Handbook. A Practical Approach to Planning and Marketing, New York: Continuum. $240 \mathrm{p}$.

GNWT - Government of the Northwest Territories (s.d), Tourism Development Handbook for the Northwest Territories, Yellowknife : GNWT, $126 \mathrm{p}$. Hazebroucq J. M. (1999). Management des projets de tourisme et de loisirs, Levallois-Perret: Gaëtan Morin éditeur. $375 \mathrm{p}$.

Langlois, G. (2006). La Coopérative de solidarité récréotouristique du Mont Adstock et son impact sur la cohésion sociale, Montréal : Centre de rechercher sur les innovations sociales, Cahiers du CRISES, Coll. «Études de cas d'entreprises d'économie sociale $\gg .80 \mathrm{p}$.

Larose, D. (2008). Tourisme, paysage et coopération, Les Journées RÉSEAU FQOLT (Fédération québécoise des organisations locales de tourisme), «Le développement durable en tourisme » (Québec: 16-17 avril 2008).

Lequin, M. (2000). «Gouvernance en écotourisme: Développement durable, développement régional et démocratie participative ». Thèse de doctorat en Études urbaines, Montréal : Université du Québec à Montréal. 523 p.

Macnulty, P. (2011). Fundamentals \& Principles of Tourism Product Development, Communication présentée au ETC-UNWTO Joint International Seminar on Tourism Product Development (Dublin, Irlande : 12-13 mai).

OMT - Organisation mondiale du tourisme (1999), Guide à l'intention des autorités locales: développement durable du tourisme, Paris: OMT. $223 \mathrm{p}$.

Pâquet, J. (2003). Outil d'aide à la décision pour classifier les secteurs d'intérêt majeurs et définir les stratégies d'aménagement pour l'intégration visuelle des coupes dans les paysages, Québec : Direction des programmes forestiers du ministère des Ressources naturelles, de la Faune et des Parcs. 15 p.

Paquette, S. et, P. Poullaouec-Gonidec (2010). Développement durable, culture et projets de territoire: portrait de démarches québécoises et étrangères exemplaires, Projet d'écotourisme ValléeBras-du-Nord (Portneuf), Chaire en paysage et environnement de l'Université de Montréal. $8 \mathrm{p}$.

Ponrouch, A. (2008). «Processus de mise en æeuvre du développement durable par les collectivités. Suiviévaluation et adaptation du SD21000 ». Thèse en Sciences de la Terre et de l'Environnement, SaintÉtienne : École Nationale Supérieure des Mines de Saint-Étienne. 544 p.

Schwab, S. (2008), Jusqu'où va la bonne gouvernance, cours COP722: séminaire en gouvernance financière Coopérative, IRECUS, cité dans LAFLEUR, M. (2008). Les Coopératives de solidarité au Québec, dossier spécial, Action nationale, février 2008. Services d'urbanisme de la MRC de Portneuf et de la municipalité de SaintRaymond

STA - Service Tourisme Attractivité (2000). Entreprendre dans le Tourisme en Pays Châtelleraudais, $16 \mathrm{p}$.

Tourisme Québec (2002). Nature et tourisme: L'écotourisme au Québec en 2002, octobre, BNQ, Table de concertation sur l'écotourisme au Québec, $<$ http://www.tourisme.gouv.qc.ca/publications/media/ 
document/etudes-statistiques/eco_QC02.pdf> consulté le 15 décembre 2011.

Tranquard, M. (2008). La conciliation des principes de développement et de bonne gouvernance au sein des Coopératives de solidarité récréo-touristiques: la Coopérative du Parc Aventures Cap Jaseux à la croisée des chemins, Chicoutimi : UQAC. 22 p.

Tranquard, M. (2013). «Opérationnalisation des projets écotouristiques : quelle ingénierie pour favoriser la durabilité environnementale? », Tourisme et territoires, Volume 3 «Performance touristique des territoires », à paraître.

Tranquard, M. et C. Gagnon (2012). Opérationnalisation des projets écotouristiques : quels critères pour favoriser la durabilité environnementale? », Téoros, vol. 31, no 2, p. 72-83.

Trillaud-Dopia, C.; J. Ruiz et S. Paquette (2010). Tourisme, paysage, et coopération dans la Vallée Bras-du-Nord, Coopérative de solidarité.

USDCEDA - U.S. Department of Commerce Economic Development Administration. (2008). Steps to Success for Rural Entrepreneurs: Starting an Ecotourism Business in Alaska.

Zimmer, P. et S. Grassmann (1996). Évaluer le potentiel touristique d'un territoire, Bruxelles : Observatoire Européen LEADER. 43 p.

\section{NOTES}

${ }^{\mathrm{a}}$ Cette recherche a été rendue possible grâce à la précieuse collaboration des gestionnaires de la Coopérative Vallée Brasdu-Nord qui ont généreusement alimenté l'auteur en données et informations en plus de se rendre disponibles pour en valider les résultats d'analyse.

${ }^{\mathrm{b}} \mathrm{Ce}$ projet a été élaboré avec l'appui du Fonds d'action québécois pour le développement durable (FAQDD).

${ }^{c}$ Etienne Beaumont, directeur adjoint, Coopérative de solidarité Vallée Bras-du-Nord: échange téléphonique du 19 février 2013.

${ }^{\mathrm{d}}$ Les principes de la Loi sur le développement durable ont d'abord trouvé leur inspiration à travers les 27 principes de développement durable issus du Sommet international de la Terre à Rio de Janeiro, au Brésil, en 1992. En 2004, basé sur la réalité québécoise en termes d'environnement, de situation sociale et d'économie, le ministère de l'Environnement a soumis 14 principes et une première ébauche d'un plan de développement durable à une consultation publique. La consultation a permis d'amender deux des principes et d'en ajouter deux autres, pour porter le total à 16 principes (source : BNQ).

ehttp://www.valleebrasdunord.com/cooperative.html rubrique Mission, objectifs et valeurs. [page consultée le 2 janvier 2013].

${ }^{\mathrm{f}}$ http://www.valleebrasdunord.com/images/to_initiative.pdf.

[page consultée le 2 janvier 2013].

${ }^{\mathrm{g}} \mathrm{http} / / /$ www.valleebrasdunord.com/co_prix.html. [page consultée le 2 janvier 2013].

hhttp://www.valleebrasdunord.com/tourisme_long.html\#long0

1 [page consultée le 2 janvier 2013]. ítoi sur le développement durable, LRQ, c D-8.1.1 (2006). Accessible notamment sur le site du MDDEP : http://www.mddefp.gouv.qc.ca/developpement/loi.htm. [page consultée le 2 janvier 2013].

${ }^{\mathrm{j}} \mathrm{L}$ 'approche BNQ 21000 est une approche générique qui propose un cadre de gestion stratégique en cohérence avec les standards internationaux et vise essentiellement à accélérer et faciliter l'intégration des seize principes de la Loi 118 sur le développement durable du Québec au sein de tous types d'organisations (source: http://www.bnq21000.qc.ca). [page consultée le 12 février 2013].

${ }^{\mathrm{k}} \mathrm{http}: / /$ www.valleebrasdunord.com/tourisme.html. [page consultée le 2 janvier 2013].

ILe contenu détaillé des quatre étapes se retrouve dans le document de présentation du projet accessible sur le site web de la Coopérative : http://www.valleebrasdunord.com/tourisme _long.html\#long01 


\section{Annexe 1 - Applications concrètes du développement durable dans le cadre du projet Tourisme, paysage et}

Principes de développement durable

1. Santé et qualité de vie :

Vie saine et productive en harmonie

2. Équité et solidarité sociale : Équité intra et intergénérationnelle, solidarité sociale avec la nature

Application au projet de la Vallée Bras-du-Nord

Rendre accessible la pratique d'activité physique (marche, canot, vélo, équitation, escalade) dans un environnement naturel exceptionnel par la beauté des paysages, la richesse de l'environnement naturel et l'aspect champêtre de la Vallée.

- Préoccupation d'assurer la pérennité du site pour les générations futures; respect des gens qui y habitent;

- Intégration des jeunes en difficulté dans notre développement touristique (projet jeunesse);

- Créer des liens entre les amateurs de plein air et la population locale;

- Application des principes coopératifs (équité et démocratie);

- Mise en œuvre d'une gestion selon la formule de la Coopérative de solidarité qui interrelie les propriétaires riverains, les travailleurs récréoforestiers et les entrepreneurs touristiques.

3. Protection de l'environnement - Réaliser un développement touristique selon les principes de l'écotourisme : en respectant l'environnement et les gens qui l'habitent, en protégeant les paysages, en élaborant et en diffusant des techniques forestières alternatives qui permettent la protection des paysages.

4. Efficacité économique $\quad$ - La vente des produits et services touristiques par la Coopérative et ses membres vise l'équilibre entre l'excellence, la rentabilité, la viabilité économique et le développement durable;

- La Coopérative innove tant dans la prestation des services et la qualité de ses aménagements que dans la gestion sur la base de la coopération;

- Son activité économique génère de l'emploi auprès d'anciens travailleurs forestiers, de jeunes en réintégration sociale, et permet de contrer l'exode des jeunes;

L'initiative diversifie l'activité économique de la région en suscitant notamment d'autres projets (agrotourisme, acériculture, PFNL).

5. Participation et engagement des citoyens et des groupes

La Coopérative regroupe une quarantaine de membres qui se sont engagés à réaliser la mission de développement harmonieux de la Vallée Bras-du-Nord. Ces membres sont composés des représentants, des villégiateurs, des agroforestiers, des travailleurs, des promoteurs touristiques, de la Corporation de développement et de la Chambre de commerce de St-Raymond. Un grand nombre d'amateurs de plein air profite des aménagements et des activités chaque année en adhérant aux principes d'écotourisme proposés. Le projet prévoit l'accroissement de leur implication. Ils seront tous sollicités à respecter les normes qui assureront la pérennité de la Vallée et de la qualité de vie des gens qui l'habitent.

6. Accès au savoir

eles outils d'information, de sensibilisation et de modes d'emploi seront élaborés (des trousses d'action), notamment afin de développer des comportements de tourisme responsable, de forestiers sensibles au paysage, de jeunes adeptes de nouvelles pratiques de l'écotourisme, de villégiateurs aguerris des modes de construction et d'aménagement en milieu naturel.

7. Pouvoir et responsabilité $\quad$ - La formule coopérative se base sur des principes démocratiques et une gestion participative des membres. Ceux-ci sont représentés au sein du CA;

- Les amateurs de plein air sont aussi informés par notre bulletin électronique (Écho de la Vallée) et par le biais de notre site web, constamment mis à jour.

8. Partenariat et coopération gouvernementale

- Participation d'Emploi Québec et de Service Canada pour les projets d'intégration des jeunes en réinsertion;

- Participation du ministère des Ressources naturelles grâce à ses subventions de mise en valeur du milieu forestier.

9. Prévention

Nous souhaitons que ce projet prévienne les coupes abusives et le déboisement par les riverains.

10. Précaution

- Elaboration de mesures de protection du territoire et des paysages. culturel, lieux et paysages

12. Préservation de la biodiversité

13. Respect de la capacité du support des écosystèmes

Protéger le caractère champêtre de la Vallée et son patrimoine bâti, notamment les fours à charbon et la chapelle du rang Saguenay.

- Coupe et mesures contrôlées du reboisement, Cueillette PFNL.

Réglementer l'activité de l'écotourisme; encadrer l'usage anarchique;

- Encadrer les activités des entreprises touristiques;

- Respecter les principes de la Coopérative;

- Respecter les normes de sécurité;

- Changer l'habitude DD (gestion déchets, recyclage);

- Entreprendre d'une façon différente des affaires en tenant compte de l'impact social et environnemental.

14. Production et consommation responsable Changement des modes d'exploitation forestière, qui tiennent compte des paysages, de la pérennité de la forêt, des cours d'eau et du caractère champêtre.

15. Pollueur-payeur

Favoriser les navettes (transport, gaz à effet de serre);

- Projet Sans trace pour les usagers.

16. Coûts

La valeur des biens et des services reflète l'ensemble des coûts occasionnés par la société durant tout

le cycle de vie, de la conception à la consommation, et à la disposition finale;

Conception des trousses d'action;

Fête champêtre. 